\title{
Forced Response of Mistuned Bladed Disks: Quantitative Validation of the Asymptotic Description
}

\author{
Oualid Khemiri* and Carlos Martel $^{\dagger}$ \\ Universidad Politécnica de Madrid, 28040 Madrid, Spain \\ and \\ Roque Corral ${ }^{\ddagger}$ \\ Industria de Turbopropulsores, S.A., 28830 Madrid, Spain
}

\begin{abstract}
The effect of small mistuning in the forced response of a bladed disk is analyzed using a recently introduced methodology: the asymptotic mistuning model. The asymptotic mistuning model is an extremely reduced, simplified model that is derived directly from the full formulation of the mistuned bladed disk using a consistent perturbative procedure based on the relative smallness of the mistuning distortion. A detailed description of the derivation of the asymptotic mistuning model for a realistic bladed disk configuration is presented. The asymptotic mistuning model results for several different mistuning patterns and forcing conditions are compared with those from a high-resolution finite element model. The asymptotic mistuning model produces quantitatively accurate results, and, probably more relevant, it gives precise information about the factors (tuned modes and components of the mistuning pattern) that actually play a role in the vibrational forced response of mistuned bladed disks.
\end{abstract}

A

$A_{a}$

$D, D_{j j^{\prime}}$

$F$

$\mathbf{F}$

$M, K$

$\mathbf{M}, \mathbf{K}$

$M_{c}, K_{c}$

$m$

N

$\mathbf{P}$

$r$

$\mathbf{X}$

$X_{j}$

$Z_{k j}$

$\Delta$

$\Delta \mathbf{M}, \Delta \mathbf{K}$

$\Delta M_{j}, \Delta K_{j}$

$\Delta M_{k}^{F}, \Delta K_{k}^{F}$

$\delta$

$\varepsilon_{j}$

$\omega$

$\omega_{a}$

$\omega_{k j}$

$\omega_{0}$

\section{Nomenclature}

$=$

$=$

$=$

$=$

$=$

$=$

$=$

$=$

$=$

$=$

$=$

$=$

$=$

$=$

$=$

$=$

$=$

$=$

$=$

$=$

$=$ coefficients

external forcing vector

stiffness matrices

number of sectors

forcing engine order

displacement vector

stiffness matrices

damping coefficient

forcing frequency traveling wave mode amplitude vector active traveling wave mode amplitude

traveling wave basis mistuning matrix

sector $j$ external forcing vector

in-sector mass and stiffness matrices

mass and stiffness matrices

sector-to-sector coupling mass and

number of degrees of freedom per sector

traveling wave modes matrix

sector $j$ displacement vector

tuned traveling wave sector $j$ mode shape

traveling wave basis mistuning matrix

mistuning mass and stiffness matrices

sector $j$ mistuning mass and stiffness matrices

$k$ Fourier harmonic mistuning mass and

in-sector mistuning amplitude

active traveling wave mode frequency

tuned traveling wave mode frequency

resonant tuned traveling wave mode frequency
*Ph.D. Candidate, Depto. de Fundamentos Matemáticos, ETSI Aeronáuticos; oualid.khemiri@upm.es.

${ }^{\dagger}$ Professor, Depto. de Fundamentos Matemáticos, ETSI Aeronáuticos; carlos.martel@upm.es.

${ }^{\ddagger}$ Department of Technology and Methods; currently Associate Professor, Department of Propulsion and Thermo Fluid Dynamics, ETSI Aeronáuticos, Universidad Politécnica de Madrid; roque.corral@itp.es.

\section{Introduction}

$\mathbf{T}$ URBOMACHINERY bladed disks are designed to have identical blades, but manufacturing processes and wear necessarily produce small differences among them. These small imperfections (known as mistuning) break the cyclic symmetry of the structure, and, despite its smallness, they can increase dramatically the forced response vibration amplitude with respect to that of the ideal case with identical blades (tuned configuration). This amplification of the response level leads to a severe reduction of the fatigue life of the blades and thus has a strong negative impact on aircraft safety, operability, and readiness.

The obvious technological relevance of mistuning forced vibration amplification has stimulated numerous studies in the past 30 years to understand mistuning effects on the dynamics of bladed disk assemblies (see, for instance, the recent presentation by Ewins [1] and the review by Castanier and Pierre [2] and exhaustive references therein).

The brute-force approach to evaluate mistuning effects is a formidable computational task. The forced response has to be computed for many different mistuning patterns, of the order of thousands, in order to statistically sample all possible distributions of imperfections. Moreover, the mistuned simulations require one to consider the whole bladed disk instead of a single sector, and therefore this kind of computation in a close to realistic configuration is extremely costly and simply not feasible in an industrial environment within the standard design timelines.

To reduce the size of the computations several simplified descriptions have been proposed. Simple mass-spring systems containing just a few degrees of freedom (DOFs) have been frequently used [38]. They qualitatively reproduce the forced response amplification induced by mistuning, but it is not clear at all how to use these simple models to produce quantitative results in a realistic configuration with a large number of DOFs. More sophisticated reduced order models (ROMs) [9-12] have also been widely used. ROMs can produce quantitatively accurate results but do not provide information about the mechanisms involved in the effect of mistuning. A reduced ROM, the fundamental mistuning model (FMM) [13-15], has been introduced in recent years. The FMM is obtained from the tuned rotor modal characteristics and focuses on a single modal family, but its applicability is limited to the description of modal families with a nearly constant vibration frequency.

More recently, a new improved methodology for mistuning analysis, referred to hereafter as the asymptotic mistuning model (AMM), has been introduced by two of the authors of this paper. 
The AMM is consistently derived from the full finite element method (FEM) formulation for the mistuned bladed disk. The derivation of the AMM relies on the application of an asymptotic perturbative procedure that is based on the relative smallness of 1) the amount of mistuning (it corresponds to a small distortion of the tuned configuration that provokes only a small variation of the natural frequencies) and 2) the damping of the tuned modes (which is typically small compared with the vibration frequency). The AMM provides a simplified description that includes only the tuned modes that are actually involved in the mistuned response, and it can be applied to any particular modal configuration. Moreover, the AMM gives also precise information about the components of the mistuning distribution that have an effect on the vibrational response of the mistuned system. This information is important for intentionally manipulating mistuning to control its effect, and it is not given by previous simplified approaches, such as ROMs and the FMM.

Previous mistuning analyses typically generate a ROM by projecting the dynamics of the system into a set of modes obtained from the separate system substructures: blade and disk. The projection performed in the derivation of the AMM uses the modes from the complete tuned system, computed using a standard FEM discretization of a single sector with cyclic boundary conditions. This idea of using the modes from the complete tuned system for the ROM construction was previously used in the subset of nominal modes (SNM) method [11] and in the component mode mistuning (CMM) method (see [16,17]). In fact, the AMM can be regarded as a particular case in which only one family of modes (or less) is considered. As a result, the CMM can successfully describe more general situations with large mistuning present (like, e.g., severe blade damage), whereas the applicability of the AMM is restricted to the case of small mistuning and damping. But, despite this relative limitation (small damping and mistuning is a quite frequent situation in practice), the AMM can give quantitatively accurate predictions of the expected amplification of the mistuned response. The main AMM contribution relies on the fact that it allows one to obtain precise information about the effect of the different components of the mistuning distribution. This kind of analysis is made possible by considering the effect of mistuning in terms of its Fourier modes, and it was not performed before in previous more complex ROM descriptions.

The AMM has been already used by the authors for the study of the optimal intentional mistuning patterns for the stabilization of aerodynamically unstable rotors [18] and to obtain accurate upper bounds on the maximum mistuned response amplification that a forced bladed disk can experience [19]. In [19] the AMM results were also successfully compared with those of a lumped system with one DOF per sector.

In this paper, we assess the AMM results against high-fidelity FEM forced response simulations of a mistuned low-pressure turbine (LPT) rotor with $\sim 50.000 \mathrm{DOFs} /$ sector and $N=84$ sectors (shown in the left plot of Fig. 1). The aim of this work is to clarify the utility of the AMM methodology as a quantitative tool for the vibration of mistuned bladed disks and also to show the relevant information that the AMM gives on the mechanisms involved in the mistuned response.

To make this paper self-contained, we begin in the next section with a short presentation of the AMM methodology, focusing on how to derive the AMM for the forced response of a general mistuned bladed disk. We then compare, in the following section, the AMM results with those obtained from a detailed FEM of a realistic mistuned rotor (Fig. 1) for two forcing configurations with engine order 9 and 27, which correspond to forcing two distinctly different modal configurations, namely, a disk dominated mode and a blade alone mode (see Fig. 2). Finally, some remarks are drawn in the Conclusions section about the accuracy of AMM and about the information that the AMM provides on the key mechanisms involved in the mistuned response.

\section{Derivation of the AMM}

In this section, we give a brief explanation of the derivation process of the AMM (see [19] for a more complete description).
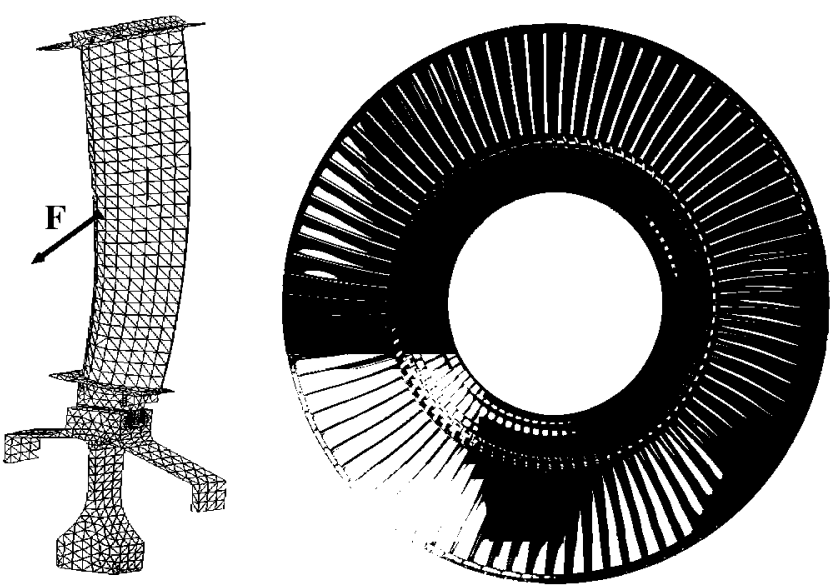

Fig. 1 FEM model of the LPT rotor sector ( $m=48.027 \mathrm{DOF} / \mathrm{sector}$ ), with the external forcing shown (left), and view of the complete rotor with $N=\mathbf{8 4}$ sectors $($ right).

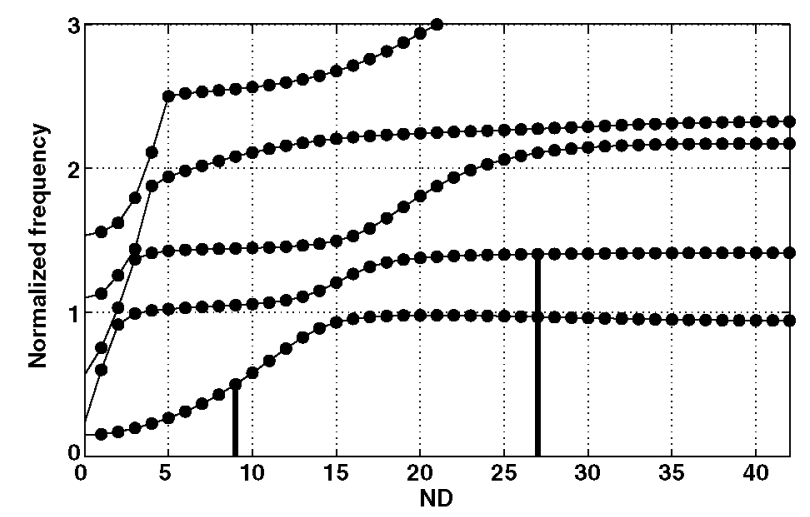

Fig. 2 Normalized frequency vs nodal diameter (ND) for the tuned rotor. Vertical lines indicate the engine order $(r=9$ and 27) for the two forcing cases considered.

The starting point is the full FEM equations for the forced oscillation of a tuned bladed disk with $N$ sectors and with $m$ independent DOFs per sector:

$$
\mathbf{K} x+\mathbf{M} \ddot{x}=f(t)
$$

The tuned mass $\mathbf{M}$ and stiffness $\mathbf{K}$ matrices are symmetric cyclic matrices given by

$$
\begin{aligned}
\mathbf{M} & =\left[\begin{array}{ccccc}
M & M_{c} & 0 & \cdots & M_{c}^{T} \\
M_{c}^{T} & M & M_{c} & \cdots & 0 \\
0 & \ddots & \ddots & \ddots & \vdots \\
\vdots & \vdots & \ddots & \ddots & M_{c} \\
M_{c} & 0 & \cdots & M_{c}^{T} & M
\end{array}\right], \\
\mathbf{K} & =\left[\begin{array}{ccccc}
K & K_{c} & 0 & \cdots & K_{c}^{T} \\
K_{c}^{T} & K & K_{c} & \cdots & 0 \\
0 & \ddots & \ddots & \ddots & \vdots \\
\vdots & \vdots & \ddots & \ddots & K_{c} \\
K_{c} & 0 & \cdots & K_{c}^{T} & K
\end{array}\right]
\end{aligned}
$$

where the diagonal components $K$ and $M$ are the $m \times m$ symmetric sector stiffness and mass matrices and the coupling between adjacent sectors is represented by the coupling stiffness and mass matrices $K_{c}$ and $M_{c}$. 
The forcing is assumed to take the form of a traveling wave (TW) excitation with frequency $\omega$ and engine order $r$ :

$$
f(t)=\mathbf{F} \cdot e^{i \omega t}+\mathrm{c} . \mathrm{c}=\left[\begin{array}{c}
F e^{i\left(\frac{2 \pi r}{N}\right) 1} \\
\vdots \\
F e^{i\left(\frac{2 \pi r}{N}\right) j} \\
\vdots \\
F e^{i\left(\frac{2 \pi r}{N}\right) N}
\end{array}\right] \cdot e^{i \omega t}+\mathrm{c} . \mathrm{c} .
$$

where c.c. stands for complex conjugate. The response displacement vector $x(t)$ has also frequency $\omega$ and can be written as

$$
x(t)=\mathbf{X} \cdot e^{i \omega t}+\mathrm{c} . \mathrm{c}=\left[\begin{array}{c}
X_{1} \\
\vdots \\
X_{j} \\
\vdots \\
X_{N}
\end{array}\right] \cdot e^{i \omega t}+\text { c.c. }
$$

with the complex vector $X_{j}$ containing the displacements of the $m$ DOF associated with sector $j$, and c.c. corresponds again to the complex conjugate.

Once we insert the expressions Eqs. (3) and (4) into Eq. (1), the forced response of the tuned system is prescribed by the linear system

$$
\left[\mathbf{K}-\omega^{2} \mathbf{M}\right] \cdot \mathbf{X}=\mathbf{F}
$$

If we now include the effect of small damping, and the mass and stiffness mistuning in the problem, the preceding system takes the more general form

$$
\left[[(1+i \delta) \mathbf{K}+\Delta \mathbf{K}]-\omega^{2}[\mathbf{M}+\Delta \mathbf{M}]\right] \cdot \mathbf{X}=\mathbf{F}
$$

where damping is considered proportional to the stiffness, and it is accounted for through the damping matrix $\delta \cdot \mathbf{K}$. The damping parameter $\delta$ is assumed to be small, $\delta \ll 1$, as it is typically the case in a realistic situation. This basically means that, after an initial excitation, the vibration performs a large number of oscillation cycles before it is damped out. The mistuning is included in the mistuning stiffness $\Delta \mathbf{K}$ and mass $\Delta \mathbf{M}$ matrices, which are of the form

$$
\begin{aligned}
\Delta \mathbf{K}= & {\left[\begin{array}{cccc}
\Delta K_{1} & 0 & \cdots & 0 \\
0 & \Delta K_{2} & \cdots & 0 \\
& & \ddots & \\
0 & 0 & 0 & \Delta K_{N}
\end{array}\right], } \\
\Delta \mathbf{M}= & {\left[\begin{array}{cccc}
\Delta M_{1} & 0 & \cdots & 0 \\
0 & \Delta M_{2} & \cdots & 0 \\
& & \ddots & \\
0 & 0 & 0 & \Delta M_{N}
\end{array}\right] }
\end{aligned}
$$

with

$$
\sum_{j=1}^{N} \Delta K_{j}=0, \quad \sum_{j=1}^{N} \Delta M_{j}=0
$$

and

$$
\Delta K_{j}=\Delta K_{j}^{T}, \quad \Delta M_{j}=\Delta M_{j}^{T} \quad \text { for } j=1 \ldots N
$$

Note that we are only considering mistuning in the in-sector matrices. The coupling matrices involve only a small subset of the DOF of the sector, and therefore its mistuning is less important than that of the main sector. The average over the $N$ sector of the mistuning distribution has been also set to zero; the average value of the mistuning distribution is equal for all sectors and can be considered, without loss of generality, as part of the tuned system.

To simplify Eq. (5) and to have a clearer understanding of the effect of the small mistuning and damping it is convenient to transform the problem into the basis of the TW natural modes of the tuned and undamped system:

$$
\mathbf{X}=\mathbf{P A}
$$

with

$$
\mathbf{P}=\frac{1}{\sqrt{N}}\left[\begin{array}{ccc}
P_{1} e^{i\left(\frac{2 \pi 1}{N}\right) 1} & \ldots & P_{N} e^{i\left(\frac{2 \pi N}{N}\right) 1} \\
\vdots & & \vdots \\
P_{1} e^{i\left(\frac{2 \pi 1}{N}\right) j} & \ldots & P_{N} e^{i\left(\frac{2 \pi N}{N}\right) j} \\
\vdots & \vdots \\
P_{1} e^{i\left(\frac{2 \pi n}{N}\right) N} & \ldots & P_{N} e^{i\left(\frac{2 \pi N N}{N}\right) N}
\end{array}\right], \quad \mathbf{A}=\sqrt{N}\left[\begin{array}{c}
A_{1} \\
\vdots \\
A_{j} \\
\vdots \\
A_{N}
\end{array}\right]
$$

where

$$
P_{k}=\left[Z_{k 1}\left|Z_{k 2}\right| \ldots \mid Z_{k m}\right]
$$

and $Z_{k 1}, \ldots, Z_{k m}$ are the complex tuned mode shapes of the $m$ TW modes with $k$ nodal diameters (i.e., IBPA $=2 \pi k / N$ ) and associated frequencies $\omega_{k 1}, \ldots, \omega_{k m}$. The tuned TW modes are the columns of $\mathbf{P}$, and the vector $\mathbf{A}$ contains their amplitudes.

In the TW basis, the tuned parts of the system Eq. (5) become diagonal and the problem simplifies to

$$
\begin{aligned}
& \left(\left[\begin{array}{ccc}
(1+i \delta) \Omega_{1}^{2}-\omega^{2} I & \ldots & 0 \\
\vdots & \ddots & \vdots \\
0 & \ldots & (1+i \delta) \Omega_{N}^{2}-\omega^{2} I
\end{array}\right]+\Delta\right)\left[\begin{array}{c}
A_{1} \\
\vdots \\
A_{N}
\end{array}\right] \\
& \quad=\left[\begin{array}{c}
0 \\
\vdots \\
P_{r}^{H} F \\
\vdots \\
0
\end{array}\right]
\end{aligned}
$$

where the diagonal matrices $\Omega_{1}, \ldots, \Omega_{m}$ contain the frequencies of the tuned TW modes with a given number of nodal diameters:

$$
\Omega_{k}^{2}=\left[\begin{array}{ccc}
\omega_{k 1}^{2} & & 0 \\
& \ddots & \\
0 & & \omega_{k m}^{2}
\end{array}\right], \quad \text { for } k=1 \ldots N
$$

and the mistuning correction matrix in the TW basis takes the form

$$
\Delta=\mathbf{P}^{H}\left(\Delta \mathbf{K}-\omega^{2} \Delta \mathbf{M}\right) \mathbf{P}
$$

Note that now, because forcing takes the form of a TW, it only has a nonzero effect in the equations corresponding to the TW modes with $k$ nodal diameters. 
The structure of the mistuning matrix in the TW basis Eq. (15) can be more easily understood if the mistuning distribution along the $N$ sectors of the structure, written in the blocks of the diagonal of $\Delta \mathbf{K}$ and $\Delta \mathbf{M}$ [see Eq. (7)], is expanded in discrete Fourier series:

$$
\begin{gathered}
\Delta K_{j}=\sum_{k=1}^{N} \Delta K_{k}^{F} e^{i\left(\frac{2 \pi k}{N}\right) j} \\
\Delta M_{j}=\sum_{k=1}^{N} \Delta M_{k}^{F} e^{i\left(\frac{2 \pi k}{N}\right) j} \quad \text { for } j=1 \ldots N
\end{gathered}
$$

The $k$ Fourier coefficients $\Delta K_{k}^{F}$ and $\Delta M_{k}^{F}$ verify [see Eqs. (8) and (9)]

$$
\begin{aligned}
\Delta K_{N}^{F} & =0, \quad \Delta M_{N}^{F}=0 \quad \text { and } \quad \Delta K_{k}^{F}=\overline{\Delta K_{-k}^{F}}, \\
\Delta M_{k}^{F} & =\overline{\Delta M_{-k}^{F}} \quad \Delta K_{k}^{F}=\left(\Delta K_{k}^{F}\right)^{T}, \\
\Delta M_{k}^{F} & =\left(\Delta M_{k}^{F}\right)^{T}, \quad \text { for } k=1 \ldots N
\end{aligned}
$$

After inserting the preceding expressions into Eq. (15), the mistuning matrix takes the form

$$
\Delta=\left[\begin{array}{cccc}
0 & \Delta_{12} & \cdots & \Delta_{1 N} \\
\Delta_{21} & 0 & \cdots & \Delta_{2 N} \\
\vdots & \vdots & \ddots & \vdots \\
\Delta_{N 1} & \Delta_{N 2} & \cdots & 0
\end{array}\right]
$$

where each block is related to the Fourier components of the mistuning by Eq. (17):

$$
\Delta_{k j}=P_{k}^{H}\left(\Delta K_{k-j}^{F}-\omega^{2} \Delta M_{k-j}^{F}\right) P_{j}
$$

Several comments regarding the structure of the mistuning matrix $\Delta$ are now in order:

1) The mistuning matrix in the TW basis is Hermitian [i.e., $\Delta=\bar{\Delta}^{T}$, recall Eq. (18)], because it corresponds to conservative elastic and inertial contributions.

2) The mistuning matrix in the TW basis [Eq. (19)] exhibits off diagonal blocks, and, thus, the effect of mistuning is to couple TWs with a different number of nodal diameters, which are completely independent in the absence of mistuning.

3) Mistuning establishes a coupling between TWs that allows the forcing with engine order $r$ to excite TWs with a number of nodal diameters different from $r$.

4) According to Eq. (20), the coupling between the TWs with $k$ and $j$ nodal diameters takes place precisely through the $(k-j)$ Fourier harmonic of the discrete Fourier transform (DFT) of the mistuning pattern.

5) Because the mistuning average has been set to zero [see Eq. (8)], $\Delta K_{N}^{F}=0$ and $\Delta M_{N}^{F}=0$, the block diagonal terms of the mistuning matrix $\Delta$ are zero.

In the mistuned problem [Eq. (13)], the damping $\delta$ is small and the mistuning matrix $\Delta$ is also small (mistuning corresponds to a small distortion of the tuned structure). Note that, in order to have the maximum response amplitude of the tuned system normalized to one, the forcing term on the right-hand side of Eq. (13) has to be of size $\delta$, and therefore it is also small.

The small values of the damping and mistuning allow us to apply an asymptotic perturbative procedure to obtain a simplified firstorder approximation of the complete mistuned problem [Eq. (13)]. Near the resonance, that is, when the forcing frequency $\omega$ (with engine order $r$ ) is close to a resonant tuned frequency $\omega_{0}$ (corresponding to a TW with $r$ nodal diameters), we can distinguish two essentially different types of TW modes:

1) The first mode is one with frequencies that are not close to $\omega_{0}$, that is, modes whose frequency $\omega_{k j}$ is such that $\left|\omega_{0}-\omega_{k j}\right|$ is large as compared with the small mistuning and damping terms. The corresponding equation for this kind of TW modes in the system Eq. (13) can be expressed as

$$
\left(\omega_{k j}^{2}-\omega^{2}\right) A_{k j}=\sum_{i, h=1}^{m}(\text { small terms }) A_{i h}+(\text { small forc. })
$$

where the coefficient on the left-hand side is large as compared with the small mistuning, damping, and forcing terms on the right-hand side because $\omega$ is close to $\omega_{0}$ and thus away from $\omega_{k j}$. After neglecting the small terms, the preceding equation yields

$$
A_{k j}=0
$$

That is, all TW modes with frequencies well apart from $\omega_{0}$ do not contribute in first approximation to the mistuned response. We call these TW modes passive modes because they do not play any role in the forced response of the mistuned system near the resonant frequency $\omega_{0}$.

2) The second mode is one with frequencies close to $\omega_{0}$, i.e., with frequencies that are at a small distance from $\omega_{0}$, of the order of the small damping and mistuning frequency corrections. We will hereafter refer to these modes as active modes because they are, in first approximation, the only ones that take part in the mistuned forced response near the resonant frequency $\omega_{0}$. After removing all passive modes of the previous item from Eq. (13), the resulting equation for an active mode with amplitude $A_{a}$ and tuned frequency $\omega_{a}$ takes the form

$$
\left(\left(\omega_{a}^{2}-\omega^{2}\right)+i \delta \omega_{a}^{2}\right) A_{a}+\sum_{a^{\prime} \neq a} \delta_{a a^{\prime}} A_{a^{\prime}}=F_{a}
$$

where the sum goes over the rest of active modes, $F_{a}=i \delta \omega_{a}^{2}$ for the active mode with $r$ nodal diameters that is being directly excited (recall that the forcing has been normalized to have tuned maximum amplitude one), and $F_{a}=0$ for the rest. All coefficients in the preceding equation are small, and, according to Eq. (20), the mistuning coupling coefficients can be written as

$$
\delta_{a a^{\prime}}=Z_{a}^{H}\left(\Delta K_{a-a^{\prime}}^{F}-\omega^{2} \Delta M_{a-a^{\prime}}^{F}\right) Z_{a^{\prime}}
$$

where $Z_{a}, Z_{a^{\prime}}, a$, and $a^{\prime}$ are, respectively, the mode shapes and the number of nodal diameters of the active modes $A_{a}$ and $A_{a^{\prime}}$. As expected, the mistuning coupling terms verify $\delta_{a a^{\prime}}=\overline{\delta_{a^{\prime} a}}$ because the stress and mass mistuning constitute a purely elastic conservative perturbation.

In a general situation, we end up with as many equations like Eq. (23) as active modes:

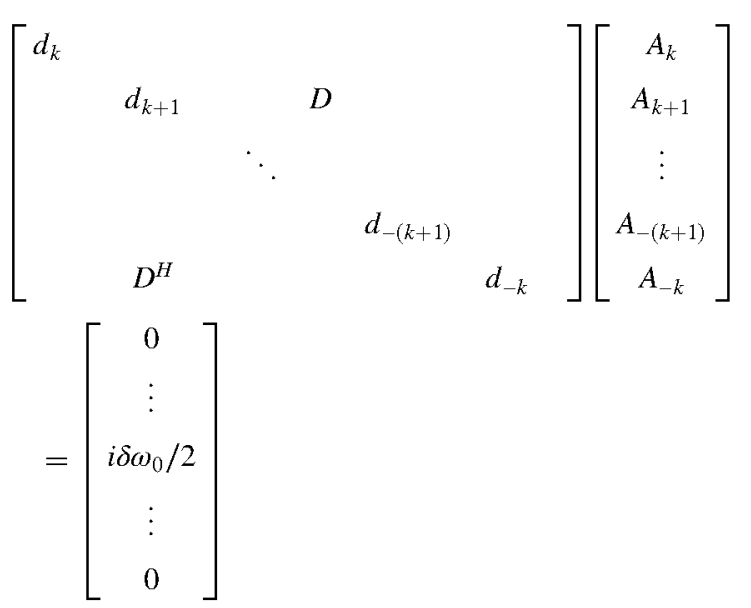

where $A_{k}, A_{k+1}, \ldots, A_{-k}$ are the amplitudes of the active TW modes and the diagonal terms are given by

$$
d_{j}=\Delta \omega_{j}-\Delta \omega+i \delta \omega_{0} / 2
$$


where $\Delta \omega_{j}=\omega_{j}-\omega_{0}, \Delta \omega=\omega-\omega_{0}, \omega$ is forcing frequency, $\omega_{0}$ is the tuned resonant frequency, $\omega_{j}$ is the frequency of the active TW with amplitude $A_{j}$, and the off diagonal elements ( $D$ in the preceding matrix) are of the form

$$
D_{j j^{\prime}}=Z_{j}^{H}\left(\Delta K_{j-j^{\prime}}^{F}-\omega_{0}^{2} \Delta M_{j-j^{\prime}}^{F}\right) Z_{j^{\prime}} /\left(2 \omega_{0}\right)
$$

Note that we have also made the following simplifications in the coefficients preceding Eqs. (26) and (27):

$$
\begin{aligned}
\omega_{j}^{2}-\omega_{0}^{2} & \sim 2 \omega_{0} \Delta \omega_{j}+\ldots, \\
\omega^{2}-\omega_{0}^{2} & \sim 2 \omega_{0} \Delta \omega+\ldots \\
\delta \omega_{j} & \sim \delta \omega_{0}+\ldots \\
\omega & \sim \omega_{0}+\ldots
\end{aligned}
$$

which are justified because the forcing frequency $\omega$ is at a small distance from the tuned frequency $\omega_{0}$, and therefore the neglected frequency variations just produce higher order corrections. It is also possible not to do these simplifications to have a more accurate approximation, but then the system for the active modes Eq. (25) becomes slightly more complicated.

We call the reduced problem given by Eq. (25) the AMM. The AMM requires one to know the tuned vibration characteristics of the system and the details of the mistuning distribution. With this information, the AMM gives an asymptotic first-order description of the response of the mistuned system when the forcing frequency is in the proximity of a tuned resonant frequency. The smaller the damping and the mistuning, the better the AMM approximation.

It is interesting to mention also that the definition of the active modes as those with frequency close to $\omega_{0}$ is not, of course, a precise quantitative definition but an asymptotic estimation. To be sure that all relevant active modes have been taken into account, the frequency band of the modes included in the AMM has to be increased until there is no significant variation on the AMM results (as will be done in Sec. III.B).

The AMM description retains only the minimal set of TW modes that are involved in the mistuning correction. The simplicity of the AMM reveals that the only TW modes that are relevant near resonance are those with frequencies near the tuned resonant frequency (i.e., the active modes) and that the effect of mistuning is to couple these modes.

The displacements are expressed in the TW basis, and this requires performing a DFT in the circumferential direction [see Eqs. (10) and (11)]. This DFT representation of the displacement, called in the literature nodal diameter spectrum, is frequently used to understand the mistuned response of the system (see, e.g., the recent paper [20]). In the AMM, the DFT of the mistuning distribution is also used [see Eqs. (16) and (17)], and, as was highlighted previously, with this DFT representation the mechanism of action of mistuning becomes clear: the coupling between the $k$ and $j$ DFT modes of the response (which are uncoupled in the tuned case) occurs precisely through the $(k-j)$ DFT mode of the mistuning.

\section{AMM Results vs High-Fidelity FEM Results}

We now compute the full mistuned forced response of the realistic LPT cantilever rotor in Fig. 1 and compare it with the AMM results. The applied excitation is a localized force acting on two elements of the sector, as is shown in Fig. 1, and we consider two forcing configurations: 1 ) engine order $r=9$ with frequency close to the first modal family and 2) engine order $r=27$ with frequency close to the second modal family (see Fig. 2). The damping coefficient is set to $\delta=0.01$, and the mistuning distributions that we use are of the form

$$
\Delta K_{j}=0, \quad \Delta M_{j}=\varepsilon_{j} M, \quad \text { for } j=1 \ldots N
$$

that is, no stiffness mistuning and mass mistuning proportional to the in-sector mass matrix. The small parameter $\varepsilon_{j}$ accounts for the mistuning distribution.
We use a preconditioned biconjugate gradient method (PBCG) [21] for the solution of the complete mistuned linear problem Eq. (5). The PBCG algorithm was simplified in our case so that it needs only one matrix-vector multiplication for each iteration instead of two. The preconditioner was constructed from the tuned stiffness matrix of each segment taking advantage of the cyclic tridiagonal structure of the full problem. The total problem size is $\mathrm{Nm} \sim 4 \cdot 10^{6}$, and it takes up to $50 \mathrm{~h}$ to compute each case of mistuned forced response using a $32 \mathrm{~Gb}$ RAM Intel Xeon at $2.83 \mathrm{GHz}$.

\section{A. Excitation with Engine Order $r=9$}

In this case, if we look close to the tuned resonant frequency of the first modal family for a forcing with engine order $r=9$, it is clearly seen from Fig. 3 that the only active modes are the TW with nine nodal diameters, which corresponds to the Fourier harmonic 9 (the one directly excited by the forcing) and its symmetric TW with Fourier harmonic -9 (not plotted in Fig. 3).

The AMM is now in its simplest possible form, with only two symmetric active TWs:

$$
\left[\begin{array}{cc}
-\Delta \omega+i \delta \omega_{0} / 2 & D \\
\bar{D} & -\Delta \omega+i \delta \omega_{0} / 2
\end{array}\right]\left[\begin{array}{c}
A_{+9} \\
A_{-9}
\end{array}\right]=\left[\begin{array}{c}
i \delta \omega_{0} / 2 \\
0
\end{array}\right]
$$

where $\Delta \omega=\omega-\omega_{0}, \omega_{0}$ is the resonant tuned frequency corresponding to the TW of the first modal family with nine nodal diameters (see Fig. 3), and the effect of mistuning is contained in the off diagonal coefficient [see Eq. (27)]:

$$
D=Z_{+9}^{H}\left(\Delta K_{18}^{F}-\omega_{0}^{2} \Delta M_{18}^{F}\right) \overline{Z_{+9}} /\left(2 \omega_{0}\right)
$$

Note that, according to the AMM approximation, the mistuned response consists of a superposition of the +9 and -9 tuned TW modes, which are coupled precisely through the $2 r=18$ Fourier

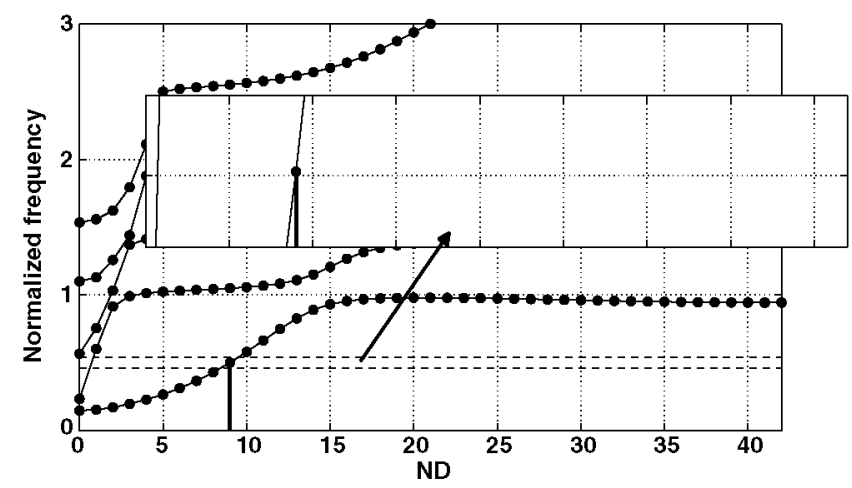

Fig. 3 Zoom of frequency vs ND plot close to the TW of the first modal family with nine nodal diameters (the zoomed region contains only one active mode).

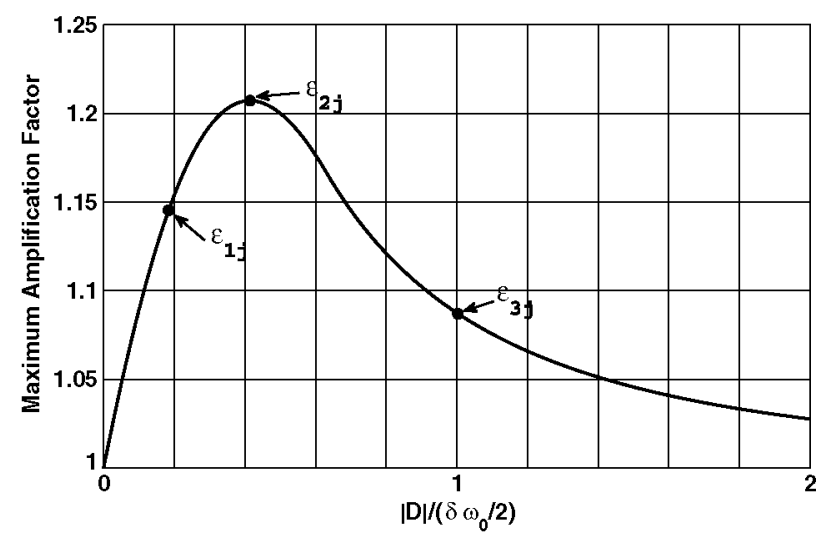

Fig. 4 Maximum attainable amplification factor vs $|D| /\left(\delta \omega_{0} / 2\right)(|D|$ is the mistuning amplitude and $\delta \omega_{0} / 2$ is the tuned TW damping). 
harmonic of the mistuning distribution, and this is the only component of the mistuning distribution that actually plays a role in the mistuning process.

The natural frequencies of the mistuned system can be computed simply by solving the eigenvalue problem obtained by setting to zero the forcing term (right-hand side) of Eq. (29). Frequency correction $\Delta \omega$ is now the eigenvalue, given by

$$
\Delta \omega= \pm|D|+i \frac{\delta \omega_{0}}{2}
$$
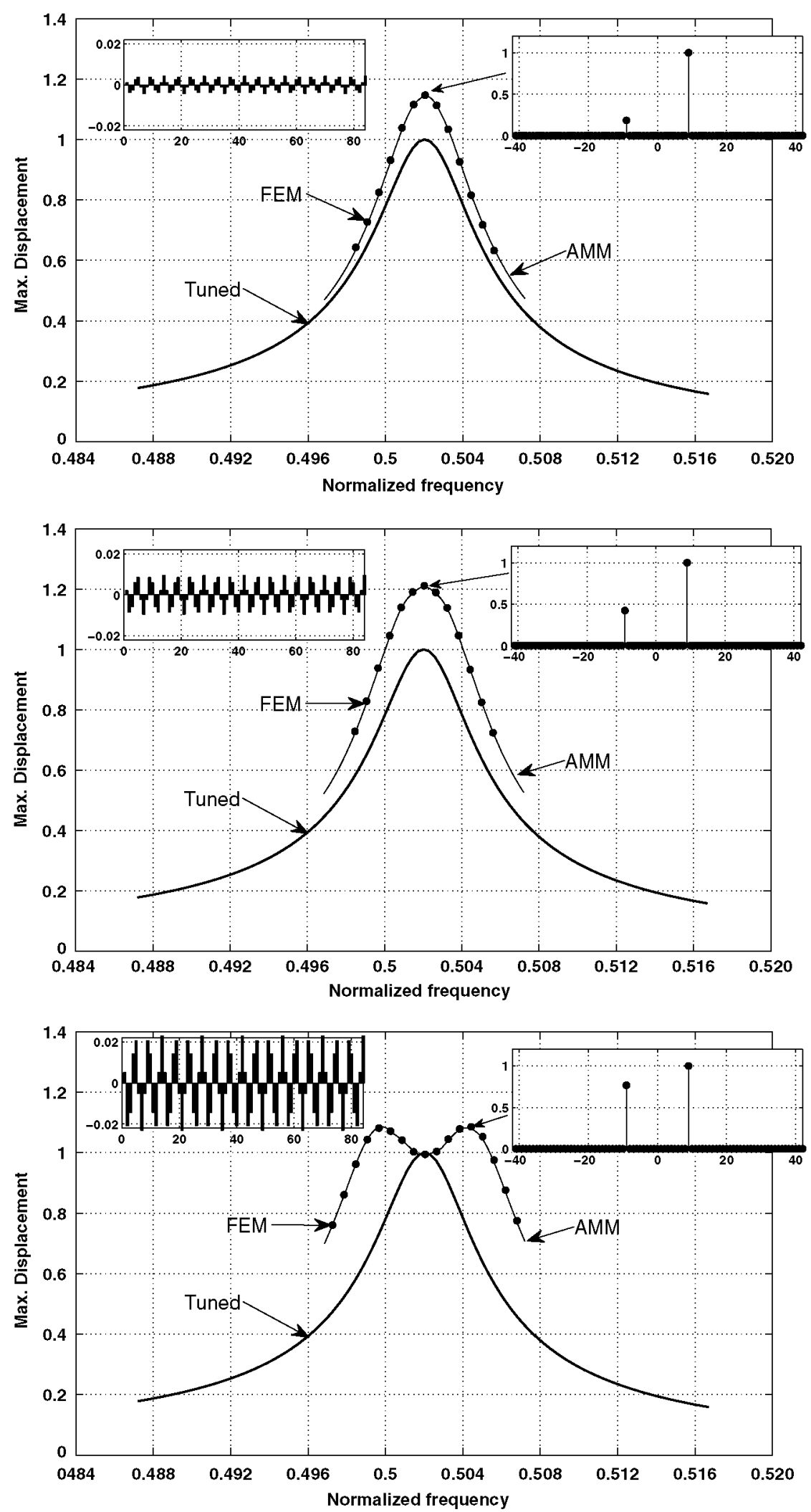

Fig. 5 Maximum response over all blades. Thick line, tuned; thin line, AMM mistuned [Eq. (29)]; dots, mistuned response computed solving the full mistuned bladed disk (Fig. 1); left inset, mistuning distribution $\varepsilon_{j}$; right inset, normalized Fourier modes of the response at the indicated point. From top to bottom, 1) $\left.\varepsilon_{1 j}=0.41 \delta \cos [2 \pi j(18) / N], 2\right) \varepsilon_{2 j}=0.94 \delta \cos [2 \pi j(18) / N]$, and 3) $\varepsilon_{3 j}=2.30 \delta \cos [2 \pi j(18) / N]$. 


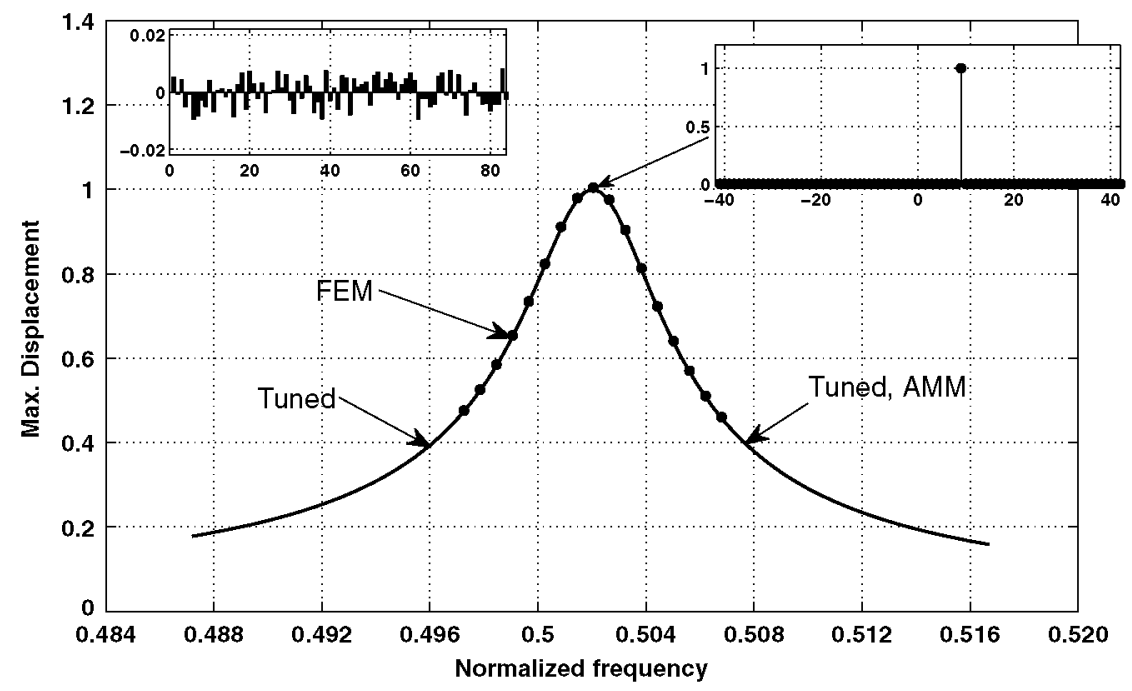

Fig. 6 Maximum response over all blades. Thick line, tuned; thin line, AMM mistuned [Eq. (29)]; dots, mistuned response computed solving the full mistuned bladed disk (Fig. 1); left inset, mistuning distribution $\varepsilon_{4 j}=0.94 \delta \operatorname{rand}(j)$; right inset, normalized Fourier modes of the response at the indicated point.

and, therefore, the small frequency splitting of the natural modes produced by mistuning is precisely $2|D|$.

Going back to the forced problem Eq. (29), this simple case can be explicitly solved, and, as was obtained in [19], the response amplitude first grows as the mistuning is increased and then decays, with a maximum amplification factor

$$
\mathrm{Amp}_{\max }=\frac{\sqrt{2}+1}{2}=1.207 \ldots
$$

The mistuned forced response amplification is plotted in Fig. 4. There is a high sensitivity of the system response to small mistuning: small values of the mistuning (comparable to the TW damping) produce an amplification of the response of $\sim 20 \%$ of the tuned amplitude. For higher values of the mistuning the amplification factor shows a slower decay, which is due to the fact that, as the mistuning grows, the splitting of the modal frequencies becomes larger, and the modes of the system no longer interact with each other. A similar amplitude magnification curve is shown in Fig. 9 of [20], where a two-DOFs/ sector lumped system is considered with a forcing engine order $r=14$ and an intentional mistuning that has only the Fourier harmonic $2 r=28$.

To check the AMM predictions we now compute the forced response of the mistuned rotor in Fig. 1 with forcing engine order $r=9$ and four different mistuning patterns:

$$
\begin{aligned}
\varepsilon_{1 j} & =0.41 \cdot \delta \cos (2 \pi j(18) / N), \\
\varepsilon_{2 j} & =0.94 \cdot \delta \cos (2 \pi j(18) / N), \\
\varepsilon_{3 j} & =2.30 \cdot \delta \cos (2 \pi j(18) / N), \\
\varepsilon_{4 j} & =0.94 \cdot \delta \operatorname{rand}(j), \quad \text { for } j=1, \ldots, N
\end{aligned}
$$

The mistuning distributions $\varepsilon_{1 j}, \varepsilon_{2 j}$ and $\varepsilon_{3 j}$ correspond, respectively, to mistuning amplitudes before, at, and after the maximum of the curve in Fig. 4, and $\varepsilon_{4 j}$ is a uniform random distribution in the range $[-1,1]$ whose Fourier mode 18 has been removed.

In Figs. 5 and 6, we compare the results obtained by solving the complete mistuned rotor [Eq. (5)] with those from the preceding AMM [Eq. (29)]. As can be appreciated from Fig. 5 the agreement is excellent. The first three mistuning patterns contain only a pure harmonic with wave number 18 . As the amplitude of the mistuning is increased we reach the maximum amplification factor (Amp $=1.207 \ldots$ ) for the mistuning distribution $\varepsilon_{2 j}$, which is precisely the worst mistuning distribution predicted by the AMM (see [19]). Additionally, if the mistuning amplitude is further increased, then the amplification factor decays, as happens for the mistuning distribution $\varepsilon_{3 j}$. Note also that, as predicted by the AMM, the response of the system contains only the Fourier mode 9 (directly excited by the forcing) and the Fourier mode -9 (see the right insets in Fig. 5), both coupled by the mistuning Fourier modes \pm 18 .

Finally, $\varepsilon_{4 j}$ is a random distribution with an amplitude similar to $\varepsilon_{2 j}$ but has no harmonic with wave number 18 (i.e., no Fourier modes \pm 18 ). Again, as predicted by the AMM, $\varepsilon_{4 j}$ has no effect on the response of the system, which behaves as if there were no mistuning at all (see Fig. 6) and shows only the directly excited Fourier mode 9 (see right inset in Fig. 6).

In summary, when the excited mode is an isolated mode, that is, when the distance of the excited tuned frequency to its neighbors is large as compared with the damping (which is typically the case for a disk dominated mode), then mistuning can increase the response amplitude of the bladed disk by a maximum factor of approximately $20 \%$. This amplification takes place for small mistuning amplitudes (of the order of the damping), and only the harmonic of the mistuning distribution with a wave number twice the engine order of the forcing has an effect on the mistuned response.

\section{B. Excitation with Engine Order $r=27$}

The applied excitation has engine order $r=27$ and frequency close to the second modal family. The situation is now more complicated than in the previous case because the resonant TW modes are not well apart from the rest of the modes. As can be clearly appreciated from Fig. 7, this is a blade dominated mode, and there are now many other TW modes with similar frequencies.

The active modes for the AMM are the tuned modes with frequency close to the excited frequency. To decide which are the

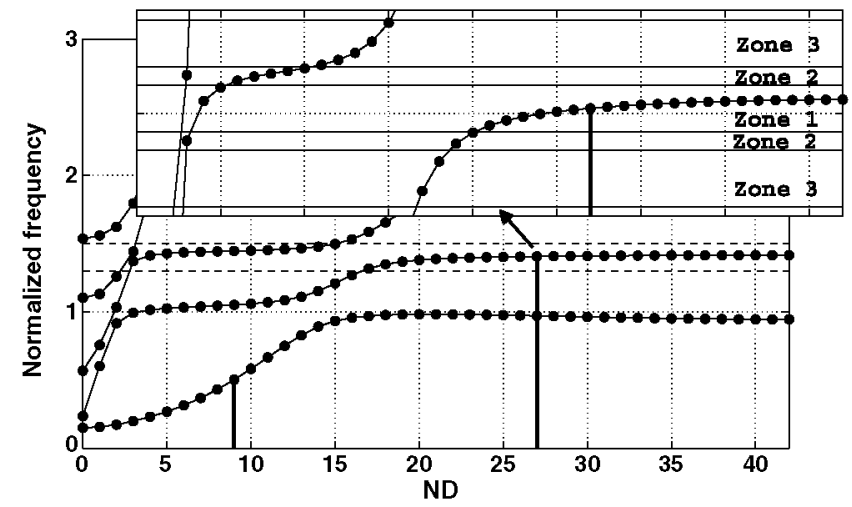

Fig. 7 Zoom of frequency vs ND plot close to the TW of the second modal family with 27 nodal diameters. 
appropriate active modes for the AMM formulation we proceed as follows: we first formulate the AMM using the active modes in zone 1 of Fig. 7, we then formulate again the AMM increasing the number of active modes to include also those in zone 2 of Fig. 7 , and then we include also those in zone 3 , and we continue increasing the number of active modes until the change in the AMM results is sufficiently small. This procedure ensures that we have taken into account all relevant active modes. This is numerically very cheap because the
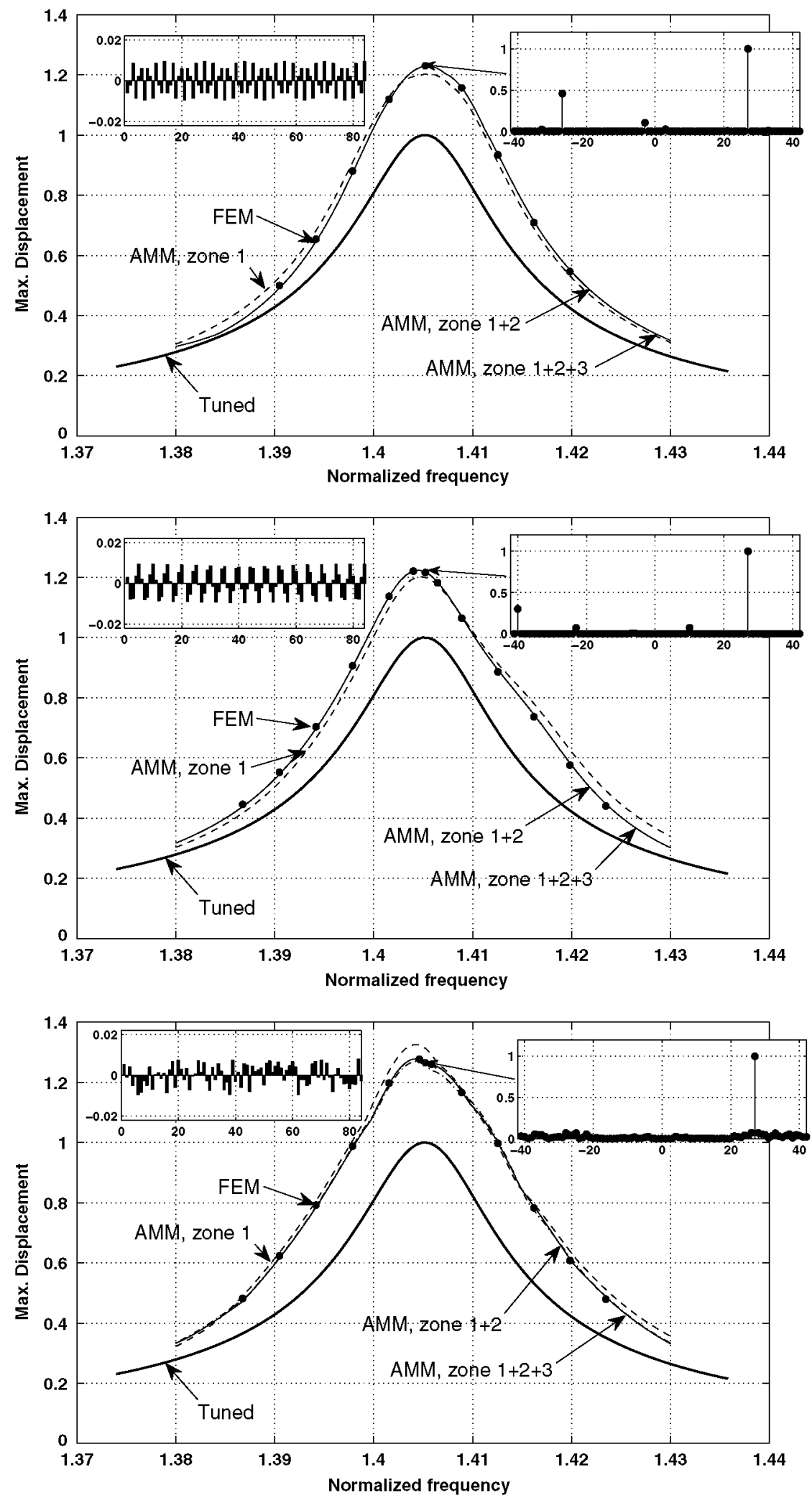

Fig. 8 Maximum response over all blades. Thick line, tuned; thin line, AMM mistuned response for active modes in zone 1 (dashed), zone $1+2$ (dashdotted), and zone $1+2+3$ (solid); dots, mistuned response computed solving the full mistuned bladed disk (Fig. 1); left inset, mistuning distribution $\varepsilon_{j}$; right inset, normalized Fourier modes of the response at the indicated point. From top to bottom, 1) $\varepsilon_{1 j}=0.94 \delta \cos [2 \pi j(54) / N]$, 2) $\varepsilon_{2 j}=0.94 \delta \cos [2 \pi j(17) / N]$, and 3$) \varepsilon_{3 j}=0.94 \delta \operatorname{rand}(j)$. 
resulting AMMs are just linear systems with size 47 for zone 1 , $47+18=65$ for zone $1+2$, and $47+18+14=79$ for zone $1+2+3$.

We present in Fig. 8 a comparison of the results from the complete mistuned rotor [Eq. (5)] with those from the AMM obtained from Eq. (25). We use three different mistuning distributions:

$$
\begin{aligned}
& \varepsilon_{1 j}=0.94 \cdot \delta \cdot \cos (2 \pi j(54) / N), \\
& \quad \varepsilon_{2 j}=0.94 \cdot \delta \cdot \cos (2 \pi j(17) / N), \quad \varepsilon_{3 j}=0.94 \cdot \delta \cdot \operatorname{rand}(j), \\
& \quad \text { for } j=1, \ldots, N
\end{aligned}
$$

two pure harmonic distributions with wave numbers 54 and 17 and a random distribution that is the same one used in the previous section.

As can be appreciated from Fig. 8, the agreement between the AMM and the FEM results is again remarkable: their difference is always below $2 \%$. This can be clearly seen in the first two plots of Fig. 8, where the results from the zone $1+2$ AMM (dash-dotted line) cannot be distinguished from those from the zone $1+2+3 \mathrm{AMM}$ (solid line), indicating that in the zone $1+2$ AMM all relevant active modes have been already considered. In the top plot of Fig. 8, the mistuning has wave number 54, and, according to the AMM predictions, the Fourier modes of the FEM response are precisely the active modes that can be excited through repeated combinations of the forced mode with the mistuning: 27 (directly forced), $27-54=-27, \quad 27+54=81=-3, \quad-27-54=-81=3$, $-3+54=51=-33$, etc. (recall that the DFT modes have to be considered modulo $N$ because $\left.e^{i\left(2^{\pi / N}\right)} \equiv e^{i(2 \pi / N)(j \pm N)}\right)$. The energy is injected at the directly forced mode, which shows the highest amplitude, and then transferred to other active modes through the coupling induced by the mistuning. The resulting amplitude of the Fourier modes decays with each combination with the mistuning.

The same happens for the second plot in Fig. 8, which corresponds to a mistuning pattern with wave number 17 , and, again according to the AMM predictions, the Fourier modes of the FEM response result from the combination with the mistuning: 27 (directly forced), $\quad 27-17=10, \quad 27+17=44=-40, \quad 10-17=-7$, $-40+17=-23$, etc. This kind of response composed of the forced mode plus the combinations with the mistuning pattern can be seen in Figs. 10 and 12 of [20], where the effect of a pure harmonic mistuning distribution is analyzed.

The random mistuning distribution in the bottom plot of Fig. 8 has many more harmonic components, and, therefore, it couples more effectively the active modes, giving rise to a response with many more Fourier modes. The need of more modes to properly represent the system response produces a slight increase in the difference between the results from the zone $1+2 \mathrm{AMM}$ and the zone $1+2+3 \mathrm{AMM}$, but this difference is still so small that we can also consider the results from zone $1+2$ AMM as sufficiently accurate.

\section{Conclusions}

The AMM $[18,19]$ as a quantitative tool for the analysis of the effect of mistuning in the resonant forced response of realistic bladed disk configurations was established.

The AMM is a simple model that is systematically derived from the complete mistuned bladed disk dynamical model. The AMM is obtained using an asymptotic perturbative procedure based on the smallness of the mistuning and the damping of the system; it has no free parameters to adjust, and it does not require one to do any a posteriori fine tuning. The AMM is built using only the tuned vibration characteristics of the system and produces accurate results of the forced response of the system for any given mistuning distribution. Regarding the computation time, the AMM can produce accurate results in a fraction of the time needed to compute the full system. For example, to compute several points (13 points) of the response curve of the first case ( $r=9$ ) one would need several days if the whole bladed disk were modeled in the simulations, but the time required to build the AMM and to compute 17 points is less than $1 \mathrm{~min}$. This reduction in the computational cost is essential for the forced response assessment of mistuned bladed disks.
The resulting AMM contains only the essential factors that actually play a role in the effect of mistuning, and it gives clear information about how mistuning operates, which can be summarized as follows:

1) The mistuned forced response of the system is composed of only those tuned TW modes that have frequency close to the tuned resonant one (the active modes).

2) The effect of the small mistuning is to couple these active TW modes.

3) The coupling between the TWs with nodal diameters $k$ and $k^{\prime}$ takes place through the Fourier harmonic of the mistuning distribution with wave number $\left(k-k^{\prime}\right)$. The Fourier harmonics of the mistuning distribution that have an effect on the response of the system are only those that can couple active modes.

4) The Fourier modes of the system response are precisely the active modes that can be excited through repeated combinations of the forced mode with the mistuning. The energy is injected at the directly forced TW, which shows the highest amplitude, and then transferred to other active modes through the coupling induced by the mistuning.

5) The expression Eq. (27) of the AMM gives precise information on how the in-sector mistuning distribution affects the response of the system, and it can be used to determine the rotor blade locations more sensitive to mistuning.

Finally, it is interesting to mention that the AMM is formally similar to the SNM [11] approach once the set of active modes is selected. Despite the similarity of the final equations, the AMM gives two essential contributions with respect to the SNM: 1) the systematic asymptotic approach, which gives information about the range of frequencies of the active modes and about the size of the small mistuning [both of the order of the damping in nondimensional variables; see Eqs. (25-27)], and 2) the unveiling of the mechanism of action of the Fourier modes of the mistuning distribution in the generation of the mistuned response.

\section{Acknowledgments}

The authors wish to thank Industria de Turbo Propulsores, S.A., for the permission to publish this paper and for its support during the project. The work of $\mathrm{OK}$ and $\mathrm{CM}$ has also been supported by the Spanish Ministerio de Educación y Ciencia (TRA2010-18054) and by the Universidad Politécnica de Madrid (GI110110039). The work of RC has also been supported by the Spanish Ministerio de Educación y Ciencia (DPI2009-14216).

\section{References}

[1] Ewins, D., "The Mistuned Blade Vibration Problem Re-Visited," Proceedings of the Twelfth International Symposium on Unsteady Aerodynamics, Aeroacoustics and Aeroelasticity of Turbomachines, Imperial College, London, U.K., Oct. 2009, pp. 323-324.

[2] Castanier, M., and Pierre, C., "Modeling and Analysis of Mistuned Bladed Disk Status and Emerging Directions," Joumal of Propulsion and Power, Vol. 22, No. 2, 2006, pp. 384-396. doi: $10.2514 / 1.16345$

[3] Sinha, A., "Computation of the Maximum Amplitude of a Mistuned Bladed Disk Assembly via Infinity Norm," Proceedings of the 1997 ASME International Mechanical Engineering Congress and Exposition, 1997, pp. 427-432.

[4] Kenyon, J., and Griffin, J., "Experimental Demonstration of Maximum Mistuned Bladed Disk Forced Response," Joumal of Turbomachinery, Vol. 125, Oct. 2003, pp. 673-681. doi: $10.1115 / 1.1624847$

[5] Kenyon, J., Griffin, J., and Feiner, D., "Maximum Bladed Disk Forced Response from Distortion of a Structural Mode," Journal of Turbomachinery, Vol. 125, No. 2, April 2003, pp. 352-363. doi: $10.1115 / 1.1540118$

[6] Whitehead, D., "Effect of Mistuning on the Vibration of Turbomachine Blades Induced by Wakes," Journal of Mechanical Engineering Science, Vol. 8, No. 1, 1966, pp. 15-21. doi:10.1243/JMES JOUR 196600800402

[7] Whitehead, D., "The Maximum Factor by Which Forced Vibration of Blades Can Increase due to Mistuning," Journal of Turbomachinery, 
Vol. 120, No. 1, Jan. 1998, pp. 115-119. doi: $10.1115 / 1.2818061$

[8] Crawley, E., and Hall, K., "Optimization and Mechanisms of Mistuning in Cascades," Journal of Engineering for Gas Turbines and Power, Vol. 107, No. 2, 1985, pp. 418-426. doi: $10.1115 / 1.3239742$

[9] Yang, M., and Griffin, J., "A Reduced Order Approach for the Vibration of Mistuned Bladed Disk Assemblies," Journal of Engineering for Gas Turbines and Power, Vol. 119, No. 1, Jan. 1997, pp. $161-167$. doi:10.1115/1.2815542

[10] Castanier, M., Ottarson, G., and Pierre, C., "A Reduced Order Modeling Technique for Mistuned Bladed-Disks," Journal of Vibration Acoustics, Vol. 119, No. 3, 1997, pp. 439-447. doi: $10.1115 / 1.2889743$

[11] Yang, M., and Griffin, J., "A Reduced Order Model of Mistuning Using a Subset of Nominal Modes," Journal of Engineering for Gas Turbines and Power, Vol. 123, 2001, pp. 893-900. doi:10.1115/1.1385197

[12] Rivas-Guerra, A., and Mignolet, M., "Maximum Amplification of Blade Response Due to Mistuning: Localization and Mode Shape Aspects of the Worst Disks," Journal of Turbomachinery, Vol. 125, No. 3, 2003, pp. 442-454. doi: $10.1115 / 1.1506958$

[13] Feiner, D., and Griffin, J., "A Fundamental Model of Mistuning for a Single Family of Modes," Journal of Turbomachinery, Vol. 124, No. 4, 2002, pp. 597-605. doi: $10.1115 / 1.1508384$

[14] Feiner, D., and Griffin, J., "Mistuning Identification of Bladed Disks Using Fundamental Mistuning Model, Part 1: Theory," Journal of Turbomachinery, Vol. 126, Jan. 2004, pp. 150-158.
[15] Feiner, D., and Griffin, J., "Mistuning Identification of Bladed Disks Using Fundamental Mistuning Model, Part 2: Application," Journal of Turbomachinery, Vol. 126, Jan. 2004, pp. 159-165.

[16] Lim, S., Bladh, R., Castanier, M. P., and Pierre, C., "A Compact, Generalized Component Mode Mistuning Representation for Modeling Bladed Disk Vibration," AIAA Paper 2003-1545, 2003.

[17] Lim, S., Bladh, R., Castanier, M. P., and Pierre, C., "Compact Generalized Component Mode Mistuning Representation for Modeling Bladed Disk Vibration," AIAA Joumal, Vol. 45, No. 9, Sept. 2007, pp. 2285-2298. doi:10.2514/1.13172

[18] Martel, C., Corral, R., and Llorens, J., "Stability Increase of Aerodynamically Unstable Rotors Using Intentional Mistuning," Joumal of Turbomachinery, Vol. 130, 2008, Paper 011005. doi:10.1115/1.2720503

[19] Martel, C., and Corral, R., "Asymptotic Description of Maximum Mistuning Amplification of Bladed Disk Forced Response," Journal of Engineering for Gas Turbines and Power, Vol. 131, 2009, Paper 022506. doi:10.1115/1.2968868

[20] Yao, J., Wang, J., and Li, Q., "Improved Modal Localization and Excitation Factors for Understanding Mistuned Bladed Disk response," Journal of Propulsion and Power, Vol. 27, No. 1, 2011, pp. 50-60. doi:10.2514/1.48244

[21] Barrett, R., Berry, M., Chan, T. F., Demmel, J., Donato, J., Dongarra, J., Eijkhout, V., Pozo, R., Romine, C., and der Vorst, H. V., Templates for the Solution of Linear Systems: Building Blocks for Iterative Methods, 2nd ed., SIAM, Philadelphia, 1994

C. Tan

Associate Editor 